\title{
Efecto del destete precoz y engorde a corral sobre la calidad de res en corderos
}

\author{
Slukwa, M.A. \\ Agencia de Extensión Rural, Instituto Nacional de Tecnología Agropecuaria (INTA), \\ Ruta $\mathrm{N}^{\circ}$ 5, km 2,5 - Corrientes, Argentina. E-mail: slukwa.mario@inta.gob.ar
}

\begin{abstract}
Resumen
Slukwa, M.A.: Efecto del destete precoz y engorde a corral sobre la calidad de res en corderos. Rev. vet. 25: 2, 135-139, 2014. El objetivo del trabajo fue comparar la calidad de las canales de corderos en lactación versus precozmente destetados y alimentados a corral con diferentes dietas. Sesenta corderos cruza Ideal de 2 meses de edad fueron aleatoriamente distribuidos en 3 grupos de 20 animales cada uno (10 machos y 10 hembras). Un grupo testigo (T) continuó lactando sobre pastura natural en tanto que los restantes se sometieron a destete precoz y alimentación a corral con dietas (isoenergéticas e isoproteicas) a base de expeller de soja (S) y girasol (G). Al momento del destete tradicional (120 días de edad) los corderos de los tres tratamientos fueron sacrificados para evaluar la calidad de res (conformación de la canal y engrasamiento). Se utilizó un diseño experimental completamente aleatorizado, siendo el animal la unidad experimental. Las medidas subjetivas de las canales fueron comparadas mediante el análisis estadístico Chi cuadrado. Los corderos del grupo $\mathrm{S}$ revelaron mayor profundidad de costilla y aumento de la compacidad de la canal, a niveles semejantes a los del grupo T. Tales índices resultaron más bajos en los animales del grupo $\mathrm{G}$.
\end{abstract}

Palabras clave: cordero, destete precoz, dietas de soja y girasol, calidad de canal.

\begin{abstract}
Slukwa, M.A.: Effect of early weaning and farmyard fattening on the lamb carcass quality. Rev. vet. 25: 2, 135-139, 2014. The objective of the trial was to compare the carcass quality of unweaned lambs versus early weaned lambs fed with different diets. Sixty lambs Ideal crossbreed, 2 months-old, were randomly distributed in 3 groups of 20 animals each (10 males and 10 females). A control group (T) continued breastfeeding while remaining animals were submitted to early weaning and fed with isoenergetic and isoproteic diets, with proteinbased soybean expeller (S) or sunflower (G). At the time of traditional weaning (120 days of age), lambs from the three treatments were slaughtered to assess the quality of carcass and fat content. A completely randomized experimental design was used, being the animal the experimental unit. Subjective measures of the carcasses were compared by means of the Chi-square statistical analysis. The lambs of group $\mathrm{S}$ revealed greater rib depth and increase of carcass compactness, at similar levels to those of the group T. Such indicators were lower in the animals of group $\mathrm{G}$.
\end{abstract}

Key words: lamb, early weaning, soybean and sunflower diets, carcass quality.

\section{INTRODUCCIÓN}

La producción de carne ovina siempre ha sido una actividad secundaria con una alta dependencia del precio internacional de la lana. Consecuentemente, en Argentina es preponderante el perfil de doble propósito (lana/carne), privilegiando razas como Corriedale, Rommey Marsh, Lincoln y Criolla (52\% del total de las

Recibido: 12 mayo 2014 / Aceptado: 16 septiembre 2014

Parte de Tesis Posgrado Prod. Anim. Subtrop. FCV-UNNE existencias ovinas del país) por sobre otras netamente carniceras como Hampshire Down y Texel. La misma tendencia se manifiesta en la zona de producción de lana de la Provincia de Corrientes (región centro-sur), donde los sistemas son mixtos extensivos, con pastoreos simultáneos del ganado bovino y ovino ${ }^{9}$.

Dentro de este esquema, se realiza un manejo reproductivo de la majada ajustando la parición y lactancia a los momentos de mayor requerimiento de la oveja, con el mejor momento en calidad y cantidad de pasto (primavera). Por tal motivo, la producción de corderos 
en la zona es zafrera, concentrada principalmente en las festividades de fin de año ${ }^{12,33}$. Recientemente ha cobrado importancia la atención hacia las características de calidad de la carne en las canales, calidad y cantidad de grasa, relación músculo-grasa, contenido en ácidos grasos y colesterol, terneza, jugosidad, $\mathrm{pH}$, olor, color y sabor de la carne de corderos de distintas razas ${ }^{4,17}$.

Es dable destacar que las características físicas, químicas y sensoriales de la carne de cordero se ven alteradas por un gran número de factores ligados al peso de sacrificio, velocidad de crecimiento y alimentación antes y después del destete ${ }^{13}$. En ovinos, la conformación de la canal tiene relación con la cantidad de músculo, si bien esta relación es baja por lo que las predicciones están sometidas a graves errores. Estudios que se han realizado en la especie ovina sobre la relación de la conformación con la cantidad de músculo de la canal parecen indicar que canales bien conformadas tienen menos carne vendible que las peores conformadas, como se deduce de los datos aportados en 1987 por la Meat and Livestock Commission ${ }^{24}$.

La mayor parte del aumento de peso de los animales es explicada por el incremento diferencial de proteínas y grasas en función del tamaño del animal, el peso, la edad y la tasa de ganancia de peso, es decir, que a mayor edad o peso o tasa de ganancia de peso, la acumulación de grasa predomina sobre la de proteína ${ }^{11}$. Distintas estrategias de alimentación podrían aumentar la deposición proteica en fases muy tempranas del crecimiento del cordero, de tal manera que las relaciones corporales y los patrones de distribución de grasa mejoren las características de la canal ${ }^{23}$.

Dado que el ritmo de crecimiento afecta los parámetros de calidad de la canal y de la carne, distintos autores utilizaron con éxito alimentos energético-proteicos para lograr un crecimiento precoz y buena co-

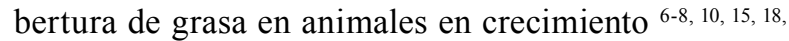
21, 26, 32. Para cumplir con las exigencias de los consumidores, no solo el productor debe cambiar, sino que la cadena agroindustrial de la carne ovina tiene que generar una respuesta que atienda a los aspectos de manejo apropiados en los establecimientos productivos, en la industria frigorífica y en los puntos de ventas. Para ello deben incentivarse las investigaciones que promuevan desarrollos tecnológicos que optimicen la oferta de carne ovina de calidad ${ }^{5}$.

No existen estándares de calidad, ni hay clasificación ni tipificación de las reses al momento de fijar los precios en el mercado interno ${ }^{1}$. Con la información adecuada, al momento de tipificar una canal ovina, en la Provincia de Corrientes se podría obtener valor agregado y generar productos diferenciados para lograr mayor competitividad en los mercados.

Atento a los antecedentes citados ut supra, el objetivo del trabajo fue evaluar las características morfométricas de las canales de corderos precozmente des- tetados y sometidos a diferentes dietas a corral, versus testigos mantenidos a campo con sus madres.

\section{MATERIAL Y MÉTODOS}

Se utilizaron 60 corderos, 30 machos y 30 hembras, cruza Ideal, nacidos entre los meses de agosto y septiembre de 2008, distribuidos en tres grupos (T, S y G) de 20 animales dada uno (diez machos y diez hembras). El grupo T estuvo compuesto por testigos al pie de madre, en tanto que los restantes fueron destetados precozmente a los 60 días de edad y alimentados a corral con dietas a base de proteína de soja (S) y girasol (G). La composición de dietas se expone en Tabla 1.

Tabla 1. Composición química promedio de los alimentos empleados.

\begin{tabular}{lcccccc}
\hline muestra & $\mathrm{H}_{2} \mathrm{O}$ & fósforo & PB & FDA & FDN & EM \\
\hline soja & 9,77 & 0,66 & 45,13 & 6,70 & & 3,01 \\
girasol & 9,61 & 1,06 & 36,90 & 27,35 & & 2,43 \\
maíz & 11,35 & 0,72 & 9,750 & 5,25 & & 3,06 \\
alfalfa & & 0,27 & 19,80 & 27,00 & 48,60 & 2,40 \\
PN inicial & & 0,14 & 14,60 & 34,90 & 57,60 & 2,22 \\
PN final & & 0,06 & 5,40 & 40,80 & 68,40 & 2,05 \\
\hline
\end{tabular}

PN: pastura natural, $\mathrm{H}_{2} \mathrm{O}$ : humedad, $\mathrm{PB}$ : proteína bruta, FDA y FDN: fibra detergente ácido y neutro, EM: energía metabolizable (en Mcal/Kg MS). Valores en \% de materia seca (excepto humedad: \%). Gentileza Laboratorio de Química, INTA Mercedes (Corrientes).

El valor nutritivo de los alimentos ofrecidos se encontró dentro de los valores promedio destinados a rumiantes. La composición proteica (PB) y energética (Mcal) de la ración con expeller de soja fue de 28,7\% $\mathrm{PB}$ y $2,8 \mathrm{Mcal} / \mathrm{Kg}$ MS respectivamente, y para la ración con expeller de girasol fue de $28,4 \%$ PB y $2,6 \mathrm{Mcal} / \mathrm{Kg}$ MS respectivamente (isoenergéticas e isoproteicas). El maíz se incluyó para balancear la energía de la dieta, en tanto que la alfalfa se empleó para el aporte de fibra. De los datos tabulados se desprende que el valor nutritivo de la pastura natural en el período de brotación en primavera, poseía un buen nivel de proteína bruta.

No ocurrió lo mismo hacia el final del ensayo, donde disminuyó su valor debido a la madurez de los pastos, mengua acompañada por la reducción de la disponibilidad de MS. A lo largo del ensayo también se observó un aumento en el contenido de FDN y FDA, revelando la pérdida de calidad del pastizal natural. Es obvia la importancia del valor nutritivo de los alimentos ofrecidos a corral, porque deben cubrir íntegramente los requerimientos de los animales encerrados.

Al finalizar el ensayo (17 de diciembre de 2008), se faenaron 45 corderos, 29 machos y 16 hembras $(10,10$ y 9 machos de los grupos S, G y T respectivamente y 6, 6 y 4 hembras de S, G y T respectivamente) para realizar su posterior análisis de calidad de res y rendimiento. Las hembras se faenaron en menor cantidad porque fueron utilizadas para la reposición de la majada. El sacrificio se realizó mediante la insensibilización y pos- 
terior sangrado de los corderos. El análisis de calidad de res y rendimiento se efectuó según una metodología oportunamente descrita ${ }^{28-30}$.

La canal fue objeto de evaluaciones objetivas y subjetivas. Las primeras se realizaron después que los animales fueron desollados, eviscerados y lavados, comenzando por la determinación del peso de la canal caliente (pcc). El rendimiento de la canal se obtuvo mediante la relación entre el (pcc) y el peso vivo (pv) a la salida del ensayo. Luego de permanecer en cámara frigorífica $24 \mathrm{~h} \mathrm{a} 4^{\circ} \mathrm{C}$ se determinó el peso de canal fría (pcf). Sobre la canal fría suspendida de los corvejones, se estimó el largo y ancho de la canal, perímetro y ancho de grupa.

Las evaluaciones subjetivas consistieron en apreciaciones visuales de la forma de las canales, que se clasificaron en base a la conformación y engrasamiento. La conformación se evaluó atendiendo al desarrollo de los perfiles de la canal, particularmente de las partes esenciales de la misma (cuartos traseros, lomo y paletilla). Para ello se utilizó una escala dividida en seis clases ( $\mathrm{S}, \mathrm{E}, \mathrm{U}, \mathrm{R}, \mathrm{O}, \mathrm{P})$ siendo cada letra una categoría distinta $(\mathrm{S}=$ superior, $\mathrm{E}=$ excelente, $\mathrm{U}=$ muy buena, $\mathrm{R}=$ buena, $\mathrm{O}=$ menos buena y $\mathrm{P}=$ inferior).

Todas estas descripciones aparecen reflejadas en los patrones fotográficos publicados por la Unión Europea (UE). El grado de engrasamiento fue evaluado mediante apreciación visual siguiendo los patrones fotográficos de la UE, que tiene en cuenta tanto la cobertura externa (grasa subcutánea) como la interna (depósitos torácico y abdominal en canales de más de $13 \mathrm{~kg}$ o sólo torácico en las de menos de $13 \mathrm{~kg}$ ). Para el grado de engrasamiento la escala utilizada fue de 1 a 5 (muy escasa; escasa; media; importante y muy importante).

Los datos obtenidos se procesaron estadísticamente a través de un diseño experimental completamente aleatorizado, siendo el animal la unidad experimental. Mediante el software SAS-8 se obtuvieron las medias aritméticas de las características morfométricas de las canales, las cuales fueron comparadas por la prueba de Chi cuadrado. Para todas las inferencias se fijó un nivel de riesgo alfa $=5 \%$, por debajo del cual se rechazó la hipótesis nula de igualdad.

\section{RESULTADOS Y DISCUSIÓN}

La Tabla 2 muestra que los rendimientos de las canales de los corderos presentaron diferencias significativas $(p<0,05)$ entre tratamientos $S$ y $G$, sin embargo, ambos grupos no lograron diferenciarse del tratamiento $\mathrm{T}$. Los menores rendimientos se presentaron en los corderos a corral con tratamiento G.

La Tabla 3 exhibe la conformación de las canales, que fueron evaluadas mediante variables cualitativas del sistema SEUROP (propuesta por la Comunidad Económica Europea CEE). Los puntajes obtenidos evidenciaron que las canales provenientes de los tratamientos a corral en su mayoría alcanzaron categorías Buena (R) y Menos Buena (O). El nivel de categoría
Tabla 2. Análisis de los rendimientos promedio de las canales.

\begin{tabular}{lcccc}
\hline grupo & PV $(\mathrm{kg})$ & pcc $(\mathrm{kg})$ & $\mathrm{pcf}(\mathrm{kg})$ & $\mathrm{R}(\%)$ \\
\hline $\mathrm{S}$ & 27,10 & 11,47 & 11,24 & $43,60 \mathrm{a}$ \\
$\mathrm{G}$ & 24,62 & 10,21 & 9,96 & $38,36 \mathrm{~b}$ \\
$\mathrm{~T}$ & 26,10 & 11,20 & 10,94 & $42,72 \mathrm{ab}$ \\
\hline
\end{tabular}

PV: peso vivo, pcc: peso de la canal caliente, pcf: peso de la canal fría, R: rendimiento. Letras diferentes en columnas indican valores que difieren estadísticamente $(p<0,05)$.

Tabla 3. Grado de conformación de las canales a las 24 h de la faena (escala CEE).

\begin{tabular}{lccccc}
\hline grupo & $\mathrm{U}$ & $\mathrm{R}$ & $\mathrm{O}$ & $\mathrm{P}$ & total \\
\hline $\mathrm{S}$ & 2 & 7 & 6 & 1 & 16 \\
$\mathrm{G}$ & - & 4 & 7 & 5 & 16 \\
$\mathrm{~T}$ & 1 & 4 & 5 & 3 & 13 \\
total & 3 & 15 & 18 & 9 & 45 \\
\hline
\end{tabular}

Tabla 4. Grado de engrasamiento de las canales a las $24 \mathrm{~h}$ de la faena (escala CEE).

\begin{tabular}{lccccc}
\hline grupo & 1 & 2 & 3 & 4 & total \\
\hline $\mathrm{S}$ & - & 2 & 13 & 1 & 16 \\
$\mathrm{G}$ & 5 & 3 & 6 & 2 & 16 \\
$\mathrm{~T}$ & 2 & 2 & 6 & 3 & 13 \\
total & 7 & 7 & 25 & 6 & 45 \\
\hline
\end{tabular}

Muy Buena (U) sólo fue logrado en dos animales del tratamiento $\mathrm{S}$ y uno del tratamiento $\mathrm{T}$. Las canales provenientes del grupo $T$ presentaron una mayor distribución entre conformaciones Muy Buena a Inferior (U-P). No se hallaron diferencias significativas entre los tratamientos $(p>0,05)$, quizás por tratarse de una categoría de animales pequeños, aún no "terminados" y con una gran variabilidad de conformaciones de la canal.

La Tabla 4 detalla el grado de engrasamiento evaluado mediante patrones fotográficos. Se observa que no existieron diferencias estadísticas entre los tratamientos ( $p>0,05)$, lo cual podría deberse a la categoría de animales faenados (corderos).

Un mayor número de animales provenientes del tratamiento $\mathrm{S}$ presentó un engrasamiento grado 3. La dispersión en el engrasamiento del testigo posiblemente haya sido consecuencia de encontrarse en un potrero grande con problemas de disponibilidad y calidad de pasto, que afectó también la producción de leche materna y la necesidad de caminar para encontrar alimento. Según especialistas en el tema, una cantidad mínima de grasa es necesaria para obtener una calidad de canal aceptable, que no se vea afectada en la cámara frigorífica. En efecto, el valor comercial de las canales de grado 1 puede disminuir en la comercialización ${ }^{28}$.

En este trabajo experimental, si bien no se encontraron diferencias $(p>0,05)$ entre tratamientos, se obtuvieron canales con una óptima deposición de grasa. No se obtuvieron reses con grado 5 que pudieran ser rechazadas en el mercado local por ser excesivamente 
Tabla 5. Medidas morfométricas de la canal entera (cm).

\begin{tabular}{lcccc}
\hline grupo & $\mathrm{B}$ & $\mathrm{Gr}$ & $\mathrm{K}$ & $\mathrm{Wr}$ \\
\hline $\mathrm{S}$ & $51,66 \mathrm{a}$ & 18,17 & 57,85 & 19,21 \\
$\mathrm{G}$ & $49,18 \mathrm{~b}$ & 19,11 & 56,68 & 18,37 \\
$\mathrm{~T}$ & $52,04 \mathrm{a}$ & 19,13 & 58,19 & 18,28 \\
\hline
\end{tabular}

B: perímetro de la grupa, Gr: ancho de grupa, K: largo de la canal, Wr: ancho de la canal. Letras diferentes en columnas indican valores que difieren estadísticamente $(\mathrm{p}<0,05)$.

Tabla 6. Medidas de la mitad izquierda de la canal (cm).

\begin{tabular}{lccccc}
\hline grupo & $\mathrm{F}$ & $\mathrm{L}$ & $\mathrm{Th}$ & $\mathrm{G} / \mathrm{F}$ & $\mathrm{PCF} / \mathrm{L}$ \\
\hline $\mathrm{S}$ & 28,57 & 50,21 & $24,17 \mathrm{a}$ & 0,699 & 0,22 \\
$\mathrm{G}$ & 29,08 & 48,83 & $22,71 \mathrm{~b}$ & 0,679 & 0,20 \\
$\mathrm{~T}$ & 28,58 & 51,92 & $23,59 \mathrm{ab}$ & 0,690 & 0,21 \\
\hline
\end{tabular}

F: largo del fémur, L: largo de media canal, Th: profundidad de la costilla, G/F: compacidad de la pierna, PCF/L: compacidad de la canal. Letras diferentes en columnas indican valores que difieren estadísticamente $(\mathrm{p}<0,05)$.

engrasadas. Sin embargo, todas tenían una capa exterior de grasa, necesaria para salvar las exigencias de la cámara frigorífica.

Sin embargo, otras investigaciones demostraron que al utilizar subproductos de soja, se mejoró la calidad de las carcasas logrando un mayor contenido de grasa de cobertura ${ }^{3,19,31}$. Esto puede ser explicado por un mejor balance energético-proteico de la dieta. Estos autores coinciden, además, en que es necesario un nivel mínimo de cobertura de grasa para mejorar la presentación general de la res, evitando el "quemado" de la carne por el frío de la cámara.

Autores australianos proponen rangos óptimos determinados por el peso de canal y el espesor de grasa, cuyos extremos van de 5-7 mm de espesor de grasa dorsal para canales entre $10-14 \mathrm{~kg}$ de peso oreado, hasta 8-14 mm para canales de 20-30 kg ${ }^{16,20}$. En el presente ensayo, las reses presentaron menor rango de engrasamiento que el obtenido por dichos autores.

En la Tabla 5 se muestran los resultados de las medidas morfométricas obtenidas externamente: largo (K) y ancho (Wr) de la canal entera, así como ancho de la grupa (Gr) y perímetro de la grupa (B). Este último grupo presentó diferencias significativas $(p<0,05)$ entre tratamientos.

Los corderos a corral que consumieron pellet de girasol $(\mathrm{G})$ también presentaron menor perímetro de grupa $(\mathrm{p}<0,05)$ que los restantes animales. La menor ganancia de peso implicaría el menor desarrollo de masa muscular detectado en esta medición, lo cual, como se detalló anteriormente, podría explicarse por las características nutritivas de la dieta ${ }^{2,27}$. Similares resultados se obtuvieron en engordes a corral, donde los corderos alimentados con girasol tuvieron menor rendimiento ${ }^{14,25}$.
En la Tabla 6 se presentan las medidas internas de la media canal izquierda, a saber: longitud de la pierna (F), longitud interna de la canal (L) y profundidad del tórax (Th) entre la $6^{a}$ y $7^{a}$ costilla. Con estos datos se calcularon los índices de compacidad de la pierna (G/F) y compacidad de la canal (PCF/L). En las mediciones realizadas, sólo se encontraron diferencias $(p<0,05)$ en la profundidad de costillas (Th). En el caso de Th los corderos alimentados a corral se diferenciaron entre sí, siendo menor en aquellos con dieta a base de girasol, aunque no se diferenciaron con el testigo.

Si bien en el presente trabajo se contrastaron dietas con diferentes fuentes proteicas, los resultados son comparables a los obtenidos en corderos usando diferentes fuentes de energía, donde se verificaron variaciones de la forma de la canal ${ }^{27}$.

Otro factor a tener en cuenta es que los índices de compacidad de la canal y de la pierna, se ven afectados por la raza y el peso vivo al sacrificio ${ }^{30}$. Es decir, es más importante el peso del animal que el índice de compacidad de la canal, pues este último será mayor al aumentar el peso de faena. La edad de la faena ejerce un efecto importante sobre la cantidad de grasa en corderos; al incrementarse la edad hay un aumento en la proporción de grasa y una disminución en la proporción de músculo. Por lo tanto, la edad del cordero es un factor fundamental a ser considerado en la comercialización y valoración de la canal ${ }^{29,30}$.

En conclusión, corderos a corral precozmente destetados y alimentados con dieta a base de expeller de soja revelaron mayor profundidad de costilla y aumento de la compacidad de la canal (a niveles semejantes a los de testigos en lactación sobre pastura natural), índices que resultaron más bajos en los animales precozmente destetados y alimentados con dieta a base de expeller de girasol.

\section{REFERENCIAS}

1. Avila J. 2007. Caracterización de la situación del sector ovino en la Provincia de Córdoba. Publ. Secretaría de Agricultura y Ganadería. Pcia. de Córdoba. Ley ovina nacional 25.422 de recuperación de la ganadería ovina. http://www.minagri.gob.ar/site/ganaderia/ley_ovina/01= presentacion/01-ley_ovina/index.php

2. Baker DH. 2000. Nutritional constrains to use soy products by animals. In: Soy in Animal Nutrition (Drackley JK, ed.), Publ. Fed. Anim. Sci. Soc., Savoy (Illinois, USA), p. 1-12.

3. Bianchi G, Garibotto G, Bentancur O. 2003. Fatty acid composition of M. Longissimus dorsi in pure and crossbred lambs in grazing systems. Ann. $49^{\text {th }}$ International Congress of Meat Science and Technology, p. 175-177. http://www.fagro.edu.uy/ agrociencia/VOL8/1/p41-50. pdf

4. Bianchi G, Garibotto G. 2004. Identificación y cuantificación de factores que afectan la calidad de carne ovina. Mem. ler. Seminario Técnico de Calidad de Carne Ovina y Vacuna, Paysandú (Uruguay), 52 p. 
5. Bianchi G, Garibotto G, Bentancur O, Feed O, Franco J, Peculio A, Sañudo C. 2005. Características productivas y calidad de la canal y carne en corderos pesados Corriedale y Hampshire Down x Corriedale. Rev Arg Prod. Anim 25: 75-91.

6. Blanchard PJ, Ellis M, Warkup CC, Hardy B, Chadwick JP, Deans GA. 1999. The influence of rate of lean and fat tissue development on pork eating quality. Anim Sci 68: 477-485.

7. Butterfield RM. 1988. New concepts of sheep growth. http://hdl.handle.net/1813/2095.

8. Byers FM. 1982. Nutritional factors affecting growth of muscle and adipose tissue in ruminants. Fed Proc 41: 2562.

9. Comisión Mixta Provincial del Ovino, Provincia de Corrientes (Argentina). 2004. Diagnóstico de la producción ovina de Corrientes. Rev IDIA XXI 7: 27-33.

10. Damergi C, Picard B, Geay Y, Robins SP. 1996. Effets de la castration précoce sur les caracteristiques du collagéne de muscles squelettiques chez le bovin male en croissance. In: Basis of the quality of typical mediterranean animal products, Ed. EAAP (Zafra, España), p. 465-470.

11. Di Marco ON. 2006. Crecimiento de vacunos para carne, Ed. INTA, Balcarce (Argentina), 204 p.

12. Flores AJ, Franz N, Rivero L, Beccaría M, Celser R, Gómez M, Flores F. 2008. Engorde de corderos a corral. INTA Mercedes. ISSN 0327-3075. http://inta.gob.ar/ documentos/at_multi_download/file/INTA_Carne\%20rumiantes.pdf

13. Gayan J, Sancha JL. 2000. Efecto del sistema de producción del cordero lechal sobre la calidad de canal y carne. Anales Curso Internacional de Genética, Centro de Investigaciones Agropecuarias Dehesón del Encinar, Toledo, España, p. 57-63.

14. Giraudo CG, Villagra SE, Cohen LE, Bidinost F, Garramuño JM. 2001. Engorde de corderos a corral en diferentes épocas del año: verano invierno. Rev Arg Prod Anim 21: 24.

15. Goliomytis M, Orfanos S, Panopoulou E, Rogdakis E. 2006. Growth curves for body weight and carcass components, and carcass composition of the Karagouniko sheep, from birth to 720 d of age. Small Rum Res 66: 222-229.

16. Hopkins DL, Adair D. 1990. Lamb carcasses produced in Zimbabwe and Australia. Wool Tech \& Sheep Breed 38: 81-82.

17. Instituto Nacional de Investigación Agropecuaria (INIA, Uruguay). 2003. Auditoria de calidad de carne ovina. Publ. INAC, Montevideo, 28 p. www.inac.gub.uy

18. Keane MG, More GJ, Connolly J. 1989. Growth and carcass composition of Friesian, Komousin x Friesian and Blonde D’Aquitaine x Friesian steers. Anim Prod 48: 353 369.
19. Kiesling DO, Swartz HA. 1997. Growth and carcass characteristics of lambs grazing cowpea, sudangrass or fed in drylot. Small Rum Res 26: 171-175.

20. Kirton AH, Johnson DL. 1979. Interrelationships between GR and other lamb carcass fatness measurements. Proceed New Zealand Soc Anim Prod 39: 194-201.

21. Korver S, Vos H, Bergstrom PL, Verstegen MW, Kleingout G. 1987. Carcass composition of veal calves dependent on genotype and feeding level. Anim Prod 45: 415421.

22. Kucseva CD, Balbuena O, Slanac AL, Schreiner JJ, Koza GA. 2002. Efecto de la suplementación con expellers de algodón, soja y girasol sobre la digestibilidad ruminal del heno y del suplemento. www.inta.gov.ar/benitez/ info/documentos/ali men/art/alimen07.htm

23. Manso T, Ruiz A, Castro T. 1998. Rendimiento de la canal, quinto cuarto y despiece de corderos de la raza Churra sometidos a distintas estrategias de alimentación. Arch Zootec 47: 73-84.

24. Meat and Livestock Commission. 1987. 488: 1287-1291. https://www.lmcni.com

25. Milicevic F, Clifton G, Cuargnolo E. 2005. Destete precoz y engorde de corderos a corral. http://www.inta.gov. ar/san tacruz/CarpetaDestetePrecoz.PDF

26. Piccard B, Micol D, Dozias D, Geay Y. 1995. Effects of compensatory growth on muscle characteristics in 2 yearold beef steers. Ann Zootech 44: 297.

27. Preziuso G, Russo C, Casarosa L, Campodoni G, Piloni S, Ciani D. 1999. Effect of diet energy source on weight gain and carcass characteristics of lambs. Small Rum Res 33: 9-15.

28. Sañudo C, Alfonso M, Sánchez A, Delfa R, Teixeira A. 2000. Carcass and meat quality in light lambs from different fat classes in the EU carcass classification system. Meat Science 56: 89-94.

29. Sañudo C. 2006. Calidad de la canal y de la carne en ovinos: factores que la determinan. Rev Arg Prod Anim 26: 155-167.

30. Sañudo, C. 2007. Quality in ruminant meat and meat products. http://www.scielo.br/scielo.php?pid=S1807$86722013000400001 \&$ script $=$ sci_arttext

31. Scerra V, Caparra P, Foti F, Lanza M, Priolo A. 2001. Citrus pulp and wheat straw silage as an ingredient in lamb diets: effects on growth and carcass and meat quality. Small Rum Res 40: 51-56.

32. Sully RJ, Morgan JH. 1982. The influence of feeding level and type of feed on the carcasses of steers. Aust $J$ Agric Res 33: 721-729.

33. Troye F. 1988. Corderos salvados/mayor porcentaje señalados. Noticias y Comentarios (INTA Mercedes, Corrientes, Argentina) No 237. 